\title{
THE BANACH-STONE PROPERTY AND THE WEAK BANACH-STONE PROPERTY IN THREE-DIMENSIONAL SPACES
}

\author{
MICHAEL CAMBERN
}

\begin{abstract}
Let $X$ and $Y$ be compact Hausdorff spaces, $E$ a Banach space, and $C(X, E)$ the space of continuous functions on $X$ to $E$. $E$ has the weak Banach-Stone property if, whenever $C(X, E)$ and $C(Y, E)$ are isometric, then $X$ and $Y$ are homeomorphic. $E$ has the Banach-Stone property if the descriptive as well as the topological conclusions of the Banach-Stone theorem for scalar functions remain valid in the case of isometries of $C(X, E)$ onto $C(Y, E)$. These two properties were first studied by $\mathbf{M}$. Jerison, and it we later shown that every space $E$ found by Jerison to have the weak Banach-Stone property actually has the Banach-Stone property, thus raising the question of whether the two properties are distinct. Here we characterize all three-dimensional spaces with the weak Banach-Stone property, and, in so doing, show the properties to be distinct.
\end{abstract}

Throughout this article $X$ and $Y$ will denote compact Hausdorff spaces, $E$ a Banach space, and $C(X, E)$ the space of continuous functions on $X$ to $E$. $\mathscr{B}(E)$ will denote the space of bounded operators on $E$, given its strong operator topology, and $C(X)$ the space of continuous functions on $X$ to the scalar field associated with $E$.

We will say that $E$ has the Banach-Stone property if, given any isometry $A$ of $C(X, E)$ onto $C(Y, E)$, there exists a homeomorphism $\tau$ of $Y$ onto $X$ and a continuous function $y \rightarrow \mathbb{Q}_{y}$ from $Y$ into $\mathscr{B}(E)$ such that, for all $y \in Y, \mathbb{Q}_{y}$ is an isometry of $E$ onto itself, and such that $(A(F))(y)=\mathbb{Q}_{y} F(\tau(y))$ for $F \in C(X, E), y \in Y$-i.e. if the Banach-Stone theorem for $C(X)$ can be completely generalized for $C(X, E)$. $E$ has the weak Banach-Stone property if the existence of an isometry $A$ of $C(X, E)$ onto $C(Y, E)$ implies that $X$ and $Y$ are homeomorphic.

The Banach-Stone and weak Banach-Stone properties were first studied in [4] by M. Jerison, who showed that every $E$ belonging to the family of strictly convex spaces has the former property, and that all spaces $E$ belonging to a larger family have the latter. The weak Banach-Stone property has also been investigated by K. Sundaresan, who showed in [6] that for every positive integer $n \geqslant 2$, the space $l_{n}^{\infty}$ fails to have the weak Banach-Stone property.

In [1] a complete characterization of all finite-dimensional Banach spaces

Received by the editors September 10, 1976 and, in revised form, April 25, 1977.

AMS (MOS) subject classifications (1970). Primary 46E15, 46B05.

(1) American Mathematical Society 1977 
with the Banach-Stone property was obtained, and in [2] that same characterization was shown to hold within the family of reflexive Banach spaces. We say that a Banach space $E$ splits if $E$ can be written as the direct sum of two nonzero subspaces $U$ and $V, E=U \oplus V$, and has norm given by $\|e\|=$ $\max \{\|u\|,\|v\|\}$ for $e=u+v \in E$. The characterization obtained in [1] and [2] is that if $E$ is a reflexive Banach space, then $E$ has the Banach-Stone property if, and only if, $E$ does not split [1, Theorems 1 and 2] and [2, Theorem].

It has also been established, [1, p. 92 and Theorem 2, p. 97], that every Banach space $E$ shown by Jerison in [4] to have the weak Banach-Stone property actually has the Banach-Stone property. Thus the question arises whether or not the two properties are indeed distinct. If $E$ is two dimensional, then it is trivially true that $E$ splits if, and only if, $E$ is isometric to $l_{2}^{\infty}$, so that by the results of [1] and [6] the properties do coincide for two-dimensional Banach spaces $E$.

In this article we characterize all three-dimensional spaces with the weak Banach-Stone property. It is shown that the only real (resp. complex) threedimensional Banach space which fails to have the weak Banach-Stone property is the real (resp. complex) space $l_{3}^{\infty}$. Now it is easy to find three-dimensional spaces which split, and yet are not isometric to $l_{3}^{\infty}$. (For example, let $U$ be a two-dimensional Hilbert space, let $V$ be the corresponding scalar field, and form $E=U \oplus V$ giving $E$ the max norm.) Since such spaces have the weak Banach-Stone property, but not the Banach-Stone property, it is a consequence of the result obtained here that the two properties are distinct.

In this article we make use of the concept of a $T$-set as introduced by $\mathrm{S}$. B. Myers in [5]. If $E$ is any Banach space, a subset $T$ of $E$ is called a $T$-set if, whenever $\left\{e_{1}, \ldots, e_{n}\right\}$ is any finite subset of $T$, then $\left\|\sum_{j=1}^{n} e_{j}\right\|=\sum_{j=1}^{n}\left\|e_{j}\right\|$, and $T$ is maximal with respect to this property. We also use I. Singer's characterization of $C(X, E)^{*}$ as the Banach space of all regular Borel vector measures $\mathbf{m}$ on $X$ to $E^{*}$, with finite variation $|\mathbf{m}|$, and norm given by $\|\mathbf{m}\|=|\mathbf{m}|(X)$, see $\left[3\right.$, p. 387]. For $x \in X, \mu_{x}$ will denote the scalar measure which is the positive unit mass concentrated at $x$, and we note for future reference that if $\varphi \in E^{*}$, then $\varphi \cdot \mu_{x} \in C(X, E)^{*}$.

Elements of $E$ will be denoted by $e, u$ and $v$ and those of $E^{*}$ by $\varphi$ and $\psi$. The value of $\varphi$ at $e$ is denoted by $\langle e, \varphi\rangle$. If $E=U \oplus V$, and if we write an element $e \in E$ as $e=u+v$, it is always implicit that $u \in U$ and $v \in V$. We denote elements of $C(X, E)$ and those of $C(Y, E)$, respectively, by $F$ and $G$, while elements of $C(X)$ and $C(Y)$ are denoted, respectively, by $f$ and $g$. The norms in $E$ and $E^{*}$ will be denoted by $\|\cdot\|$, while norms in $C(X, E)$, $C(Y, E), C(X)$ and $C(Y)$ are denoted by $\|\cdot\|_{\infty}$.

If $E \neq\{0\}$ is a Banach space, and $T$ is a $T$-set in $E$, then there exists an element $\varphi \in E^{*}$ with $\|\varphi\|=1$ such that $\langle e, \varphi\rangle=\|e\|$ for $e \in T$, [1, Proposition 1]. Let $\mathscr{F}=\left\{\varphi \in E^{*}\right.$ : $\|\varphi\|=1$ and $\langle e, \varphi\rangle=\|e\|$ for all $e$ belonging to 
some $T$-set $T \subseteq E$ \}. If $E$ is finite dimensional, then by a $T$-basis for $E^{*}$ we mean a basis consisting of elements of $\mathscr{F}$. Such bases always exist, [1, Proposition 2]. The following lemma is easily established.

Lemma 1. Let $E$ be a Banach space that splits, $E=U \oplus V$. Then $U^{*}$ is isometrically isomorphic to $V^{0}$, the annihilator of $V$ in $E^{*}$, under the map which sends an element $\varphi \in U^{*}$ to that element $\varphi^{\prime} \in V^{0} \subseteq E^{*}$ defined by $\left\langle e, \varphi^{\prime}\right\rangle=$ $\langle u, \varphi\rangle$, for $e=u+v \in E$.

Henceforth, we will cease to distinguish, in notation, between an element of $U^{*}$ and its image under the isometry of the lemma. The same symbol $\varphi$ may denote both an element of $U^{*}$ and its image in $V^{0}$ under the above correspondence.

Lemma 2. Let $E$ be a Banach space that splits, $E=U \oplus V$. Then given $a$ $T$-set $T$ in $U$, the set $\mathbf{T}=\{u+v \in E: u \in T$ and $v$ is an element of $V$ with $\|v\| \leqslant\|u\|\}$ is a $T$-set in $E$.

Proof. One readily verifies that norm is an additive function on finite subsets of $\mathbf{T}$. We show that $\mathbf{T}$ is maximal in $E$ with respect to this property.

If $\mathbf{T}$ were not maximal, there would exist an element $e_{0}=u_{0}+v_{0} \in E-$ $\mathbf{T}$ such that $\left\|e_{0}+e\right\|=\left\|e_{0}\right\|+\|e\|$ for all $e \in \mathbf{T}$. Since $e_{0} \notin \mathbf{T}$, either (i) $\left\|v_{0}\right\|>\left\|u_{0}\right\|$, or (ii) $\left\|v_{0}\right\| \leqslant\left\|u_{0}\right\|$ but $u_{0} \notin T$.

If (i) holds, choose $u \in T$ such that $\|u\|=\left\|v_{0}\right\|-\left\|u_{0}\right\|$. (This is possible, since $T$ is a cone, [5, Lemma 2.1, p. 133].) Then $u \in \mathbf{T}$ and $\left\|e_{0}+u\right\|=\left\|v_{0}\right\|$ $<\left\|v_{0}\right\|+\|u\|=\left\|e_{0}\right\|+\|u\|$, contradicting the fact that $e_{0}$ must add in norm with every element of $\mathbf{T}$.

If (ii) holds, again by [5, Lemma 2.1] there exists an element $u \in T$ such that $\left\|u_{0}+u\right\|<\left\|u_{0}\right\|+\|u\|$. Then $u \in \mathbf{T}$. We have

$$
\left\|e_{0}\right\|+\|u\|=\left\|u_{0}\right\|+\|u\|>\left\|u_{0}+u\right\|
$$

and

$$
\left\|e_{0}\right\|+\|u\|=\left\|u_{0}\right\|+\|u\| \geqslant\left\|v_{0}\right\|+\|u\|>\left\|v_{0}\right\|,
$$

so that $\left\|e_{0}+u\right\|=\max \left\{\left\|u_{0}+u\right\|,\left\|v_{0}\right\|\right\}<\left\|e_{0}\right\|+\|u\|$, and we again reach a contradiction. The proof of the lemma is thus complete.

As an immediate consequence of Lemmas 1 and 2 we have the following:

LEMMA 3. If $\varphi \in U^{*},\|\varphi\|=1$, and $\langle u, \varphi\rangle=\|u\|$ for all $u$ belonging to some $T$-set $T \subseteq U$, then considered as an element of $V^{0} \subseteq E^{*},\langle e, \varphi\rangle=\|e\|$ for all $e$ belonging to some $T$-set $\mathbf{T} \subseteq E$.

LEMMA 4. Let $E$ be a three-dimensional Banach space that splits, $E=U \oplus$ $V$, where $U$ is two dimensional. Suppose that $U$ does not split, and let $\left\{\varphi_{1}, \varphi_{2}\right\}$ be a $T$-basis for $U^{*}=V^{0} \subseteq E^{*}$. If $A$ is an isometry of $C(X, E)$ onto $C(Y, E)$ then for each $x \in X$ we have

$$
A^{*-1}\left(\varphi_{i} \cdot \mu_{x}\right)=\varphi_{i}^{\prime} \cdot \mu_{y}, \quad i=1,2,
$$


where the $\varphi_{i}^{\prime}$ are elements of $E^{*}$ with $\left\|\varphi_{i}^{\prime}\right\|=1$, and $y$ is an element of $Y$ which depends only on $x$.

Proof. By Lemma 3 and [1, Lemma 2, p. 94], for each $x \in X$ and $i=1,2$, $A^{*-1}\left(\varphi_{i} \cdot \mu_{x}\right)$ is of the form $\varphi_{i}^{\prime} \cdot \mu_{y_{i}}$, where $\left\|\varphi_{i}^{\prime}\right\|=1$. We want to show that for fixed $x$ we have $y_{1}=y_{2}$.

Suppose, to the contrary, that for some $x \in X$, we have $y_{1} \neq y_{2}$. Then since $U$ does not split, and $\left\{\varphi_{1}, \varphi_{2}\right\}$ is a basis for $U^{*}$, by [1, Lemma 1, p. 93] there exists a $T$-set $T$ in $U$ and a $\varphi \in U^{*}=V^{0}$ with $\|\varphi\|=1$, such that $\langle u, \varphi\rangle=$ $\|u\|$ for all $u \in T$, and such that both of the sets $\left\{\varphi, \varphi_{1}\right\}$ and $\left\{\varphi, \varphi_{2}\right\}$ are linearly independent.

By Lemma 3, $\langle e, \varphi\rangle=\|e\|$ for all $e$ belonging to some $T$-set $\mathbf{T}$ in $E$, so that by [1, Lemma 2], $A^{*}\left(\varphi \cdot \mu_{x}\right)$ is of the form $\varphi^{\prime} \cdot \mu_{y}$ for some $\varphi^{\prime} \in E^{*}$ with $\left\|\varphi^{\prime}\right\|=1$, and some $y \in Y$. Let $I$ be the subset of $\{1,2\}$ such that for $i \in I$, the support of $A^{*-1}\left(\varphi_{i} \cdot \mu_{x}\right)$ is equal to $y$. Then $I$ is either empty or a set containing one element.

We wish to show that $\left\{\varphi^{\prime}, \varphi_{i}^{\prime}: i \in I\right\}$ is a linearly independent subset of $E^{*}$. This is trivially true if $I=\varnothing$, so suppose that $I$ is a singleton, $I=\left\{i_{0}\right\}$, and suppose that there exists a scalar $\lambda$ such that $\varphi^{\prime}=\lambda \varphi_{i_{0}}^{\prime}$. Then

$$
\varphi^{\prime} \cdot \mu_{y}-\lambda \varphi_{i_{0}}^{\prime} \cdot \mu_{y}=0
$$

in $C(Y, E)^{*}$, and hence

$$
A^{*}\left(\varphi^{\prime} \cdot \mu_{y}-\lambda \varphi_{i_{0}}^{\prime} \cdot \mu_{y}\right)=\varphi \cdot \mu_{x}-\lambda \varphi_{i_{0}} \cdot \mu_{x}=0
$$

in $C(X, E)^{*}$. Thus $\varphi=\lambda \varphi_{i_{0}}$ in $E^{*}$, contradicting the fact that $\left\{\varphi, \varphi_{i_{0}}\right\}$ is a linearly independent set. Hence $\left\{\varphi^{\prime}, \varphi_{i}^{\prime}: i \in I\right\}$ is linearly independent as claimed.

Thus we can take a vector $e \in E$ such that $\left\langle e, \varphi_{i}^{\prime}\right\rangle=0, i \in I$, but $\left\langle e, \varphi^{\prime}\right\rangle$ $\neq 0$. Let $g$ be any element of $C(Y)$ with $g(y) \neq 0$, and such that the support of $g$ is disjoint from the support (or supports) of $A^{*-1}\left(\varphi_{i} \cdot \mu_{x}\right)$ for $i \notin I$, and define $G \in C(Y, E)$ by $G\left(y^{\prime}\right)=g\left(y^{\prime}\right) \cdot e, y^{\prime} \in Y$. Since $\varphi \in U^{*}$ and $\left\{\varphi_{1}, \varphi_{2}\right\}$ is a basis for $U^{*}$, there exist scalars $\lambda_{i}$ such that $\varphi+\lambda_{1} \varphi_{1}+\lambda_{2} \varphi_{2}=$ 0 . Thus $\varphi \cdot \mu_{x}+\lambda_{1} \varphi_{1} \cdot \mu_{x}+\lambda_{2} \varphi_{2} \cdot \mu_{x}=0$ in $C(X, E)^{*}$, and so

$$
\begin{aligned}
0 & =\int A^{-1}(G) d\left(\varphi \cdot \mu_{x}+\lambda_{1} \varphi_{1} \cdot \mu_{x}+\lambda_{2} \varphi_{2} \cdot \mu_{x}\right) \\
& =\int G d\left(A^{*-1}\left(\varphi \cdot \mu_{x}+\lambda_{1} \varphi_{1} \cdot \mu_{x}+\lambda_{2} \varphi_{2} \cdot \mu_{x}\right)\right) \\
& =\int G d\left(\varphi^{\prime} \cdot \mu_{y}\right)+\sum_{i \in I} \lambda_{i} \int G d\left(\varphi_{i}^{\prime} \cdot \mu_{y}\right) \\
& =\left\langle G(y), \varphi^{\prime}\right\rangle+\sum_{i \in I} \lambda_{i}\left\langle G(y), \varphi_{i}^{\prime}\right\rangle \\
& =g(y)\left\langle e, \varphi^{\prime}\right\rangle \neq 0 .
\end{aligned}
$$

This contradiction completes the proof of the lemma.

TheOREM. Let $E$ be a three-dimensional Banach space. Then E fails to have 
the weak Banach-Stone property if, and only if, $E$ is isometric to $l_{3}^{\infty}$.

Proof. The "if" part of the theorem has been established by Sundaresan, [6, p. 22]. Hence we assume that $E$ is not isometric to $l_{3}^{\infty}$ and show that $E$ has the weak Banach-Stone property.

If $E$ does not split, then $E$ has the Banach-Stone property, and we are done. Thus suppose that $E$ splits, $E=U \oplus V$, where $U$ is two dimensional and $V$ is one dimensional. Now $U$ cannot split, for otherwise $E$ would be isometric to $l_{3}^{\infty}$, contrary to the hypothesis.

Let $A$ be an isometry of $C(X, E)$ onto $C(Y, E)$, and let $\left\{\varphi_{1}, \varphi_{2}\right\}$ be a $T$-basis for $U^{*}=V^{0} \subseteq E^{*}$. Then for $x \in X$, define $\tau(x)=y$ if the supports of $A^{*-1}\left(\varphi_{i} \cdot \mu_{x}\right)$ are equal to $y$, for $i=1,2$. By Lemma $4, \tau$ is a well-defined function from $X$ to $Y$.

We wish to show, first of all, that $\tau$ is one-one. Suppose, to the contrary, that $x_{1}, x_{2}$ are distinct points of $X$, but that $\tau\left(x_{1}\right)=\tau\left(x_{2}\right)=y$. This would mean that $A^{*-1}\left(\varphi_{i} \cdot \mu_{x_{j}}\right)$ is of the form $\varphi_{i j} \cdot \mu_{y}$ for $i=1,2$ and $j=1,2$. Since $E^{*}$ is three dimensional, $\left\{\varphi_{i j}: i=1,2\right.$ and $\left.j=1,2\right\}$ is a linearly dependent set, so that there exist scalars $\alpha_{i j}$, not all zero, such that $\sum_{i, j \in\{1,2\}} \alpha_{i j} \varphi_{i j}=0$. Then $\Sigma_{i, j \in\{1,2\}} \alpha_{i j} \varphi_{i j} \cdot \mu_{y}=0$ in $C(Y, E)^{*}$, so that

$$
A^{*}\left(\sum_{i, j \in\{1,2\}} \alpha_{i j} \varphi_{i j} \cdot \mu_{y}\right)=\sum_{i, j \in\{1,2\}} \alpha_{i j} \varphi_{i} \cdot \mu_{x_{j}}=0
$$

in $C(X, E)^{*}$. We may suppose, without loss of generality, that $\alpha_{11} \neq 0$. Since $\varphi_{1}$ and $\varphi_{2}$ are linearly independent, there exists an $e \in E$ with $\left\langle e, \varphi_{2}\right\rangle=0 \neq$ $\left\langle e, \varphi_{1}\right\rangle$. Take $f \in C(X)$ with $f\left(x_{2}\right)=0 \neq f\left(x_{1}\right)$, and define $F \in C(X, E)$ by $F\left(x^{\prime}\right)=f\left(x^{\prime}\right) \cdot e$, for $x^{\prime} \in X$. We would then have

$$
\begin{aligned}
0 & =\int F d\left(\sum_{i, j \in\{1,2\}} \alpha_{i j} \varphi_{i} \cdot \mu_{x_{j}}\right) \\
& =\alpha_{11}\left\langle F\left(x_{1}\right), \varphi_{1}\right\rangle+\alpha_{21}\left\langle F\left(x_{1}\right), \varphi_{2}\right\rangle+0 \\
& =\alpha_{11} f\left(x_{1}\right)\left\langle e, \varphi_{1}\right\rangle \neq 0,
\end{aligned}
$$

which is absurd. Thus $\tau$ is one-one, as claimed.

We next show that $\tau$ maps $X$ onto $Y$. As above, we assume the contrary is true, and arrive at a contradiction. Suppose that $y_{1} \in Y-\tau(X)$. By Lemma 4 and [1, Lemma 2], with $A^{*}$ replacing $A^{*-1}$, we would have $A^{*}\left(\varphi_{j} \cdot \mu_{y_{1}}\right)$ is of the form $\psi_{j} \cdot \mu_{x}, j=1,2$, for some point $x \in X$ dependent on $y_{1}$. Now $\tau(x)=y_{2}$, where by our assumption, $y_{2}$ cannot be equal to $y_{1}$. This means that $A^{*-1}\left(\varphi_{i} \cdot \mu_{x}\right)=\varphi_{i}^{\prime} \cdot \mu_{y_{2}}, i=1,2$, where the $\varphi_{i}^{\prime}$ are elements of norm one in $E^{*}$. Since $\left\{\varphi_{1}, \varphi_{2}, \psi_{1}, \psi_{2}\right\}$ is a linearly dependent set in $E^{*}$, there are scalars $\lambda_{i}, \alpha_{j}$, not all zero, such that $\sum_{i, j \in\{1,2\}} \lambda_{i} \varphi_{i}+\alpha_{j} \psi_{j}=0$, and thus

$$
\sum_{i, j \in\{1,2\}} \lambda_{i} \varphi_{i} \cdot \mu_{x}+\alpha_{j} \psi_{j} \cdot \mu_{x}=0
$$

in $C(X, E)^{*}$. Hence 


$$
\begin{aligned}
A^{*-1}\left(\sum_{i, j \in\{1,2\}} \lambda_{i} \varphi_{i} \cdot \mu_{x}+\alpha_{j} \psi_{j} \cdot \mu_{x}\right) \\
=\lambda_{1} \varphi_{1}^{\prime} \cdot \mu_{y_{2}}+\lambda_{2} \varphi_{2}^{\prime} \cdot \mu_{y_{2}}+\alpha_{1} \varphi_{1} \cdot \mu_{y_{1}}+\alpha_{2} \varphi_{2} \cdot \mu_{y_{1}}=0
\end{aligned}
$$

in $C(Y, E)^{*}$. If we note that since $\varphi_{1} \cdot \mu_{x}$ and $\varphi_{2} \cdot \mu_{x}$ are linearly independent elements of $C(X, E)^{*}, \varphi_{1}^{\prime} \cdot \mu_{y_{2}}$ and $\varphi_{2}^{\prime} \cdot \mu_{y_{2}}$ are linearly independent in $C(Y, E)^{*}$, and thus $\varphi_{1}^{\prime}$ and $\varphi_{2}^{\prime}$ are linearly independent in $E^{*}$, a construction exactly analogous to that of the preceding paragraph then yields an element $G \in C(Y, E)$ such that

$$
\int G d\left(\lambda_{1} \varphi_{1}^{\prime} \cdot \mu_{y_{2}}+\lambda_{2} \varphi_{2}^{\prime} \cdot \mu_{y_{2}}+\alpha_{1} \varphi_{1} \cdot \mu_{y_{1}}+\alpha_{2} \varphi_{2} \cdot \mu_{y_{1}}\right) \neq 0 .
$$

This contradiction thus establishes that $\tau$ is onto.

Finally, we show that $\tau$ is continuous. Suppose, to the contrary, that there exists a net $\left\{x_{\beta}: \beta \in B\right\}$ in $X$ such that $x_{\beta} \rightarrow x_{0}$, but that $y_{\beta}=\tau\left(x_{\beta}\right) \not \rightarrow \tau\left(x_{0}\right)$ $=y_{0}$. Then there is a compact neighborhood $N$ of $y_{0}$ such that for every $\beta_{0} \in B$, there is a $\beta \geqslant \beta_{0}$ such that $y_{\beta}$ lies outside $N$. Now by [1, Lemma 2] and by the definition of $\tau, A^{*-1}\left(\varphi_{1} \cdot \mu_{x_{0}}\right)=\varphi_{1,0} \cdot \mu_{y_{0}}$ for some $\varphi_{1,0} \in E^{*}$ with $\left\|\varphi_{1,0}\right\|=1$. Fix $e_{0} \in E$ with $\left\|e_{0}\right\|=1$, such that $\left\langle e_{0}, \varphi_{1,0}\right\rangle=1$. Choose $g_{0} \in$ $C(Y)$ with $1=\left\|g_{0}\right\|_{\infty}=g_{0}\left(y_{0}\right)$, and such that the support of $g_{0}$ is contained in $N$. Then define $G_{0} \in C(Y, E)$ by $G_{0}(y)=g_{0}(y) \cdot e_{0}$, for $y \in Y$. We have

$$
\begin{aligned}
\left\langle\left(A^{-1}\left(G_{0}\right)\right)\left(x_{0}\right), \varphi_{1}\right\rangle & =\int A^{-1}\left(G_{0}\right) d\left(\varphi_{1} \cdot \mu_{x_{0}}\right) \\
& =\int G_{0} d\left(A^{*-1}\left(\varphi_{1} \cdot \mu_{x_{0}}\right)\right)=\int G_{0} d\left(\varphi_{1,0} \cdot \mu_{y_{0}}\right) \\
& =\left\langle G_{0}\left(y_{0}\right), \varphi_{1,0}\right\rangle=\left\langle e_{0}, \varphi_{1,0}\right\rangle=1 .
\end{aligned}
$$

Now since $x_{\beta} \rightarrow x_{0}$ and $\left\langle\left(A^{-1}\left(G_{0}\right)\right)(x), \varphi_{1}\right\rangle$ is a continuous function of $x \in X$, there exists a $\beta_{0} \in B$ such that if $\beta \geqslant \beta_{0}$ then $\operatorname{Re}\left\langle\left(A^{-1}\left(G_{0}\right)\right)\left(x_{\beta}\right), \varphi_{1}\right\rangle>\frac{1}{2}$. Thus fix a $\beta \geqslant \beta_{0}$ such that $y_{\beta}=\tau\left(x_{\beta}\right)$ lies outside $N$. Again we have $A^{*-1}\left(\varphi_{1} \cdot \mu_{x_{\beta}}\right)=\varphi_{1, \beta} \cdot \mu_{\gamma_{\beta}}$, for some $\varphi_{1, \beta} \in E^{*}$ with $\left\|\varphi_{1, \beta}\right\|=1$. Choose an $e_{\beta} \in E$ with $\left\|e_{\beta}\right\|=1$ such that $\left\langle e_{\beta}, \varphi_{1, \beta}\right\rangle=1$. Then take an element $g_{\beta}$ of $C(Y)$ with $1=\left\|g_{\beta}\right\|_{\infty}=g_{\beta}\left(y_{\beta}\right)$, and such that the support of $g_{\beta}$ is disjoint from $N$. Define $G_{\beta} \in C(Y, E)$ by $G_{\beta}(y)=g_{\beta}(y)$. $e_{\beta}$, for $y \in Y$. Now $G_{0}$ and $G_{\beta}$ both have norm one and they have disjoint supports, so that $\left\|G_{0}+G_{\beta}\right\|_{\infty}=1$. However,

$$
\begin{aligned}
&\left\|A^{-1}\left(G_{0}+G_{\beta}\right)\right\|_{\infty} \geqslant\left\|\left(A^{-1}\left(G_{0}\right)\right)\left(x_{\beta}\right)+\left(A^{-1}\left(G_{\beta}\right)\right)\left(x_{\beta}\right)\right\| \\
& \geqslant \operatorname{Re}\left[\left\langle\left(A^{-1}\left(G_{0}\right)\right)\left(x_{\beta}\right), \varphi_{1}\right\rangle+\left\langle\left(A^{-1}\left(G_{\beta}\right)\right)\left(x_{\beta}\right), \varphi_{1}\right\rangle\right] \\
&> \frac{1}{2}+\operatorname{Re} \int A^{-1}\left(G_{\beta}\right) d\left(\varphi_{1} \cdot \mu_{x_{\beta}}\right) \\
&=\frac{1}{2}+\operatorname{Re} \int G_{\beta} d\left(A^{*-1}\left(\varphi_{1} \cdot \mu_{x_{\beta}}\right)\right)=\frac{1}{2}+\operatorname{Re} \int G_{\beta} d\left(\varphi_{1, \beta} \cdot \mu_{y_{\beta}}\right) \\
&=\frac{1}{2}+\operatorname{Re}\left\langle G\left(y_{\beta}\right), \varphi_{1, \beta}\right\rangle=\frac{1}{2}+\operatorname{Re}\left\langle e_{\beta}, \varphi_{1, \beta}\right\rangle=\frac{3}{2},
\end{aligned}
$$


which contradicts the fact that $A^{-1}$ is norm-preserving. Hence $\tau$ is a continuous, one-one map of $X$ onto $Y$, and is thus a homeomorphism.

\section{REFERENCES}

1. M. Cambern, On mappings of spaces of functions with values in a Banach space, Duke Math. J. 42 (1975), 91-98.

2. __ Reflexive spaces with the Banach-Stone property, Rev. Roumaine Math. Pures Appl. (to appear).

3. N. Dinculeanu, Vector measures, Pergamon Press, New York, 1967.

4. M. Jerison, The space of bounded maps into a Banach space, Ann. of Math. (2) 52 (1950), 309-327.

5. S. B. Myers, Banach spaces of continuous functions, Ann. of Math. (2) 49 (1948), 132-140.

6. K. Sundaresan, Spaces of continuous functions into a Banach space, Studia Math. 48 (1973), 15-22.

Department of Mathematics, University of California, Santa Barbara, California 93106 